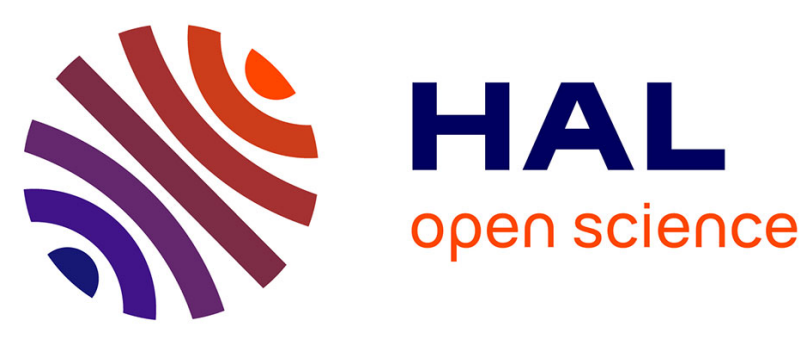

\title{
L'atelier de la Manutention
}

Armand Desbat, Anne Schmitt

\section{To cite this version:}

Armand Desbat, Anne Schmitt. L'atelier de la Manutention. Gallia - Archéologie de la France antique, 1997, Les productions des ateliers de potiers antiques de Lyon (2e partie) : les ateliers du Ier s. après J.-C., 54, pp.45-50. 10.3406/galia.1997.3234 . halshs-00980652

\section{HAL Id: halshs-00980652 \\ https://shs.hal.science/halshs-00980652}

Submitted on 29 Jan 2020

HAL is a multi-disciplinary open access archive for the deposit and dissemination of scientific research documents, whether they are published or not. The documents may come from teaching and research institutions in France or abroad, or from public or private research centers.
L'archive ouverte pluridisciplinaire HAL, est destinée au dépôt et à la diffusion de documents scientifiques de niveau recherche, publiés ou non, émanant des établissements d'enseignement et de recherche français ou étrangers, des laboratoires publics ou privés.

\section{(ㅇ)(1) $\$$}

Distributed under a Creative Commons Attribution - NonCommercial - NoDerivatives 44.0 


\title{
L'ATELIER DE LA MANUTENTION
}

\author{
Armand DesBat et Anne SchmitT
}

Résumé. Des trataux réalisés én 1966 dans le termain de la Manutention militaire, quai Saint-Vincent, ont rénélé un four et des témoins de la production de mortiers et d'amphores associés à des résidus de four de vervier: L'élude du maténiel permel de situer cefle production entre 50 et 100 apres J.-C.

\begin{abstract}
In 1966, a building site in the plot of la Manutention, on Saint-Vincent quay close to the site of la Muetle, gave evidence of a kiln and production of mortarias and amphoras, with remains of a glassuonerer kiln. The study of the material shozus that the production must be dated in the second part of the l" century.
\end{abstract}

\section{(II) \\ IRCONSTANCES DES DÉCOUVERTES}

Le domaine de la Manutention militaire, autre5.). S Manutention des Vivres, quai Sainte-Maricgux-Chaînes, est situé aujourd'hui quai Saint-Vincent, en val de la place de la Butte et en amont du site de la Muette (fig. 15). A. Comarmond y signale quelques découvertes sporadiques au XIX's. (Comarmond, 18551857).

Durant l'été 1966, le creusement d'une fosse, dans l'enceinte de la Manutention militaire pour l'installation d'un réservoir de carburant, fut l'occasion de nouvelles observations réalisées par A. Grange et M. Picon. Dans cette fosse de $7 \mathrm{~m} \mathrm{x} \mathrm{3,5} \mathrm{m} \mathrm{sur} \mathrm{2,30} \mathrm{m}$ de profondeur apparurent un four de potier et des couches de rebuts de fabrication de céramique et de verrerie.

I.e four de potier circulaire, d'un diamètre interne de 1,40 m, présentait une partie de sa sole conservée, supportée par une voûte de tegulae; l'alandier de $90 \mathrm{~cm}$ de long pour une section de $35 \mathrm{~cm} \times 50 \mathrm{~cm}$ était obturé par une dalle en pierre.

Dans la partie basse du four ont été recueillis cinq vases à parois fines intacts de forme ovoïde, pouvant laisser supposer une production comparable à celle de la

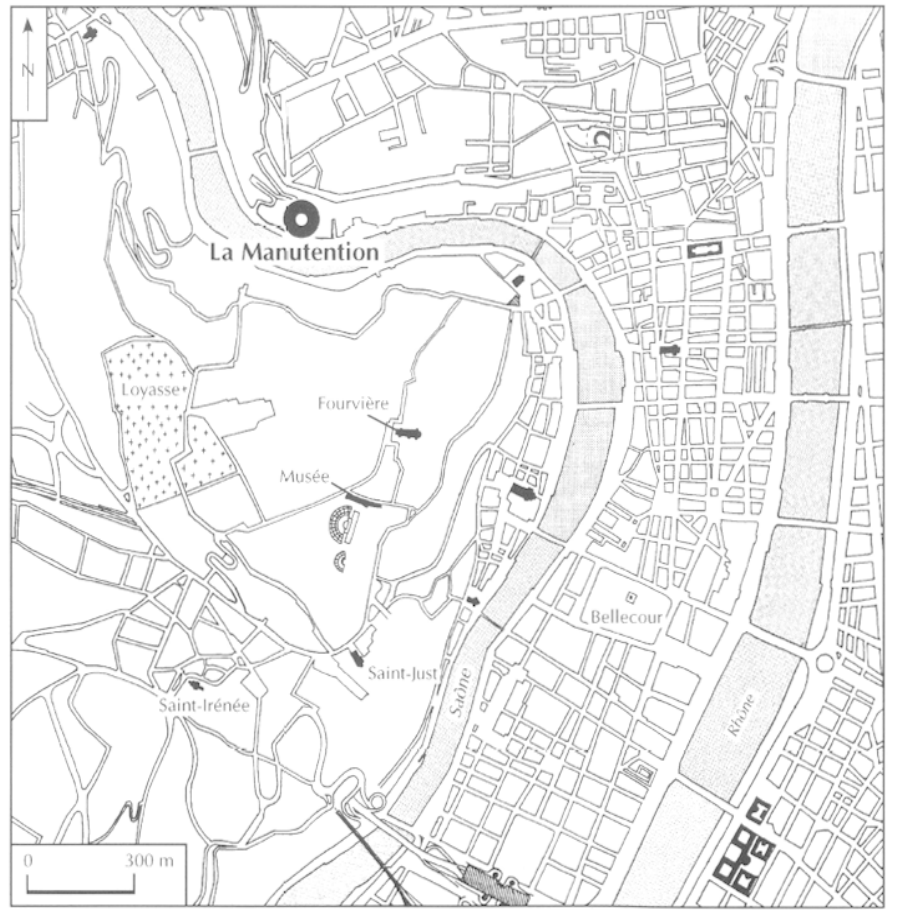

Fig. 15. Plan de localisation de l'atelier de la Mamutention.

Butte $^{6}$. Toutefois les couches de remblais antiques ont livré les résidus d'autres productions. Le matériel

6. Ce matériel décrit par A. Grange n'a pas été retrouvé. 
recucilli est peu abondant, compte tenu de la surface réduite de la fouille, mais atteste la production à proximité d'amphores et de morticrs.

A. D.

\section{LES PRODUCTIONS}

\section{LES MORTIERS}

Plusicurs lèvres de mortiers ont été récoltées. Elles présentent des profils variés.

- Mortiers à lèvre en bandeau. Il s'agit de mortiers avec un rebord haut, très évasé sur un exemplaire. Ils constituent l'évolution tardive du mortier à bandeau classique de l'époque augustéenne (fig. 16, n" 1 et 2).

- Mortiers à lèvre formant un marli. Le marli presque horizontal se recourbe légèrement. Ce modèle de mortier peu courant se rencontre rarement sur les sites lyonnais. Il se distingue des mortiers à lèvere retombante qui deviennent fréquents durant la seconde moitić du I" $\mathrm{s}$. (fig. 16, n" 3 et 4).

- Mortiers à lève triangulaire (fig. 16, n"5). Ce dernier type, pratiquement inconnu sur les sites de consommation lyonnais, n'est représenté que par cet exemplaire.
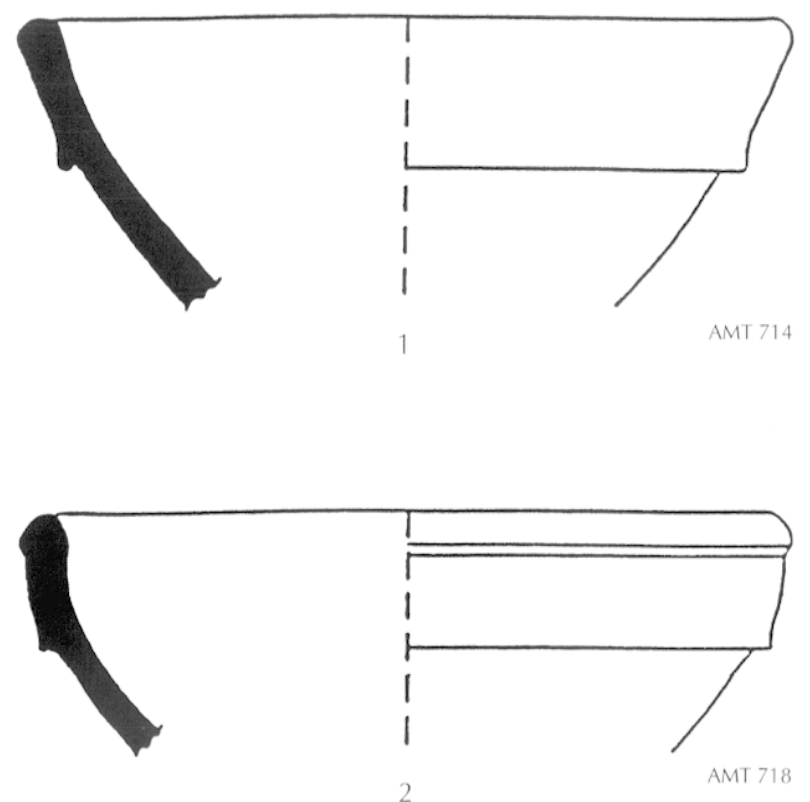

Fig. 16. Les mortiers de l'atelier de la Manutention (échelle : 1/4).
Malgré leur diversité de forme, tous les exemplaires recueillis présentent le même type de pâte de couleur beige à dégraissant sableux.

\section{LES AMPHORES}

I.es amphores trouvées en association avec les mortiers présentent des pâtes d'aspect identique. Comme pour les mortiers, les amphores présentent une assez grande diversité dans le profil des lèvres.

\section{LES AMPHORES À SAUMURE}

Ces amphores, qui constituent l'essentiel du matériel, sont représentées par des cols, des lèvres et des fonds.

Les cols peuvent se classer en quatre groupes.

- Col avec lèvre à ressaut : trois exemplaires se classent dans ce groupe qui correspond au type $3 \mathrm{~A}$, également présent dans l'atelier de la Muette (pl. 12, n"s 1 à 3).

- Col évasé à lèvre arrondie : ce groupe qui ne compte que deux lèvres constitue une variante du type 3 B (pl. 12, $n^{\prime \prime} 4$ et 5).

- Col évasé à lèvre triangulaire : quatre cols appartiennent à ce groupe qui constitue une autre variante du type 3B (pl. 12, n" 6 à 9).
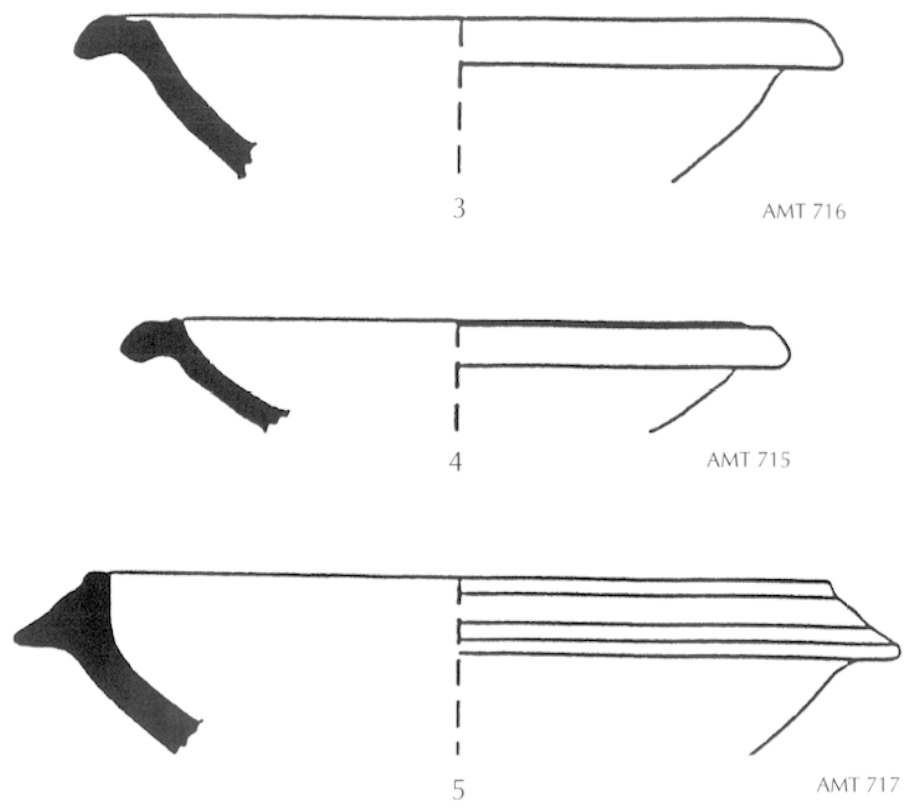
- Col évasé à ressaut légèrement marqué et lèvre arrondie : il s'agit d'amphores de petit module représentées par deux excmplaires qui ne sont pas identiques (pl. 12, $\mathrm{n}^{\circ 1} 10$ ct 11$)$.

Un col et une anse ne peuvent être attribués précisément à l'un de ces groupes mais appartiennent à des amphores à saumure (pl. 12, n" 12 et 13).

I.es fonds sont eux-mêmes de formes variées. Deux pilons creux appartiennent sans conteste à des amphores de type 3 (pl. 12, n" 14 et 15). Deux autres pilons creux se rattachent à d'autres formes. L'un (pl. 12, n"16) présente un léger ressaut qui le rapproche des pilons de Dressel 2/4 (type 2B), toutefois ceux-ci sont généralement pleins. Le second pilon (pl. 12, n"17), également creux, ne présente pas de ressaut et est légèrement plus évasć. Ces deux pilons évoquent des amphores fusclées. Pcut-être s'agit-il de pilons de Dressel 16, autre type d'amphores à garum dont la forme se rapproche des Dresscl $2 / 4$.

\section{LES AMPHORES À FOND PLAT}

Deux fragments, une lèvre ct un fond, appartiennent à un type d'amphore à fond plat proche du type Dressel 28 dont ils pourraient constituer une imitation locale (fig. $17, \mathrm{n}^{\mathrm{N}} 1$ et 2 ).

Un dernier type est représenté par une anse d'une forme particulière. Celle-ci, étroite et longue, présente

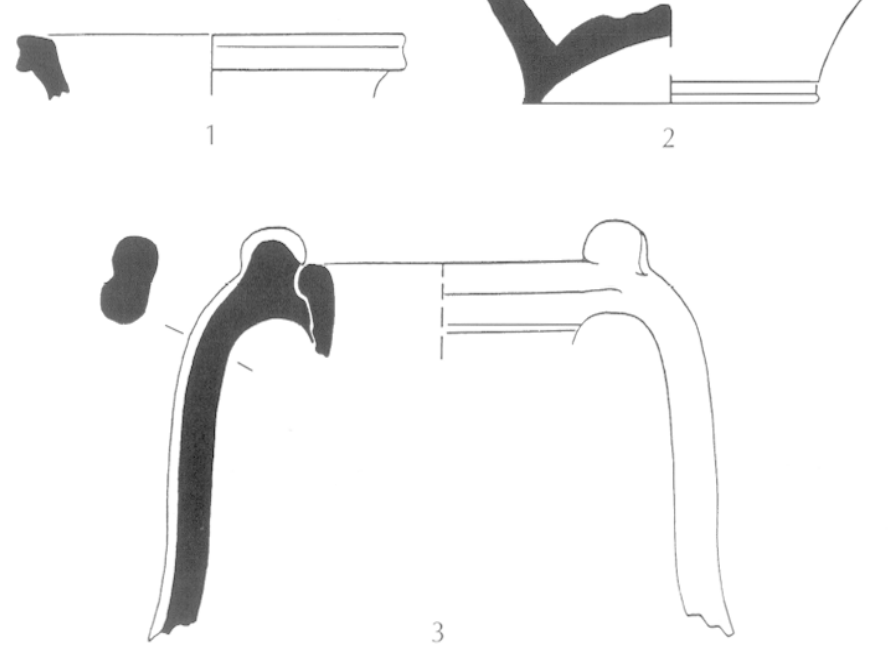

Fig. 17. Les amphores à fond plal de l'atelier de la Manutention (échelle : 1/4). un sillon et deux protubérances au sommet. Elle se rattache à une lèvre arrondic, soulignée par un léger ressaut. La taille de l'anse et l'ćpaisseur des parois, de même que le type de pâte avec dégraissant sableux, suggèrent qu'il s'agit d'unc petite amphore, mais celle-ci n'ćvoque rien de connu (fig. 17, n"3).

\section{LES DÉCOUVERTES ANCIENNES}

Il faut ajouter à cette découverte celle de trois amphores complètes recucillies au XIX ${ }^{\prime}$ s. et actuellement conservées au Musée de la Civilisation galloromaine.

Ia première (Comarmond, 1855-1857, n" 5) est une Dressel 2/4, appartenant au type 2B : le n" 5 (fig. 18, n"1) est une amphore à col cylindrique, rétréci à la base, avec une lèvre épaisse en bourrelet. Une estampille circulaire illisible figure sur le col. Les anses sont pseudo-bifides. L'épaule est très carénée. Le pied plein comporte le ressaut caractéristique.

Les deux autres (n"631 et 633) appartiennent au type $3 \mathrm{~A}:$ le n" 631 est une amphore avec col à ressaut, le pilon est creux (fig. 18, n"2); le n"633, bien qu'appartenant au même type, se distingue du type précédent par quelques détails : le ressaut sous la lèvre en bourrelet est moins marqué et le pilon est plein (fig. 18, n”3).

A. D.

\section{CARACTÉRISTIQUES CHIMIQUES DES PRODUCTIONS}

L'étude des compositions chimiques et pétrographiques des céramiques communes découvertes sur le site de la Manutention a porté sur 19 exemplaires de mortiers et amphores (Dressel 2/4 et Dressel 9).

Les compositions chimiques de ces céramiques sont très proches de celles des céramiques communes grossières de la Muette, l'approvisionnement en argiles alluviales devait être peu éloigné pour les deux ateliers. Deux exemplaires ont une teneur en chaux relativement faible $(14 \%)$ et leur composition chimique est très proche de celle du groupe A' de la Muette. Les autres exemplaires ont une pâte très calcaire, leur composition chimique moyenne est donnée dans le tableau II. Ce 

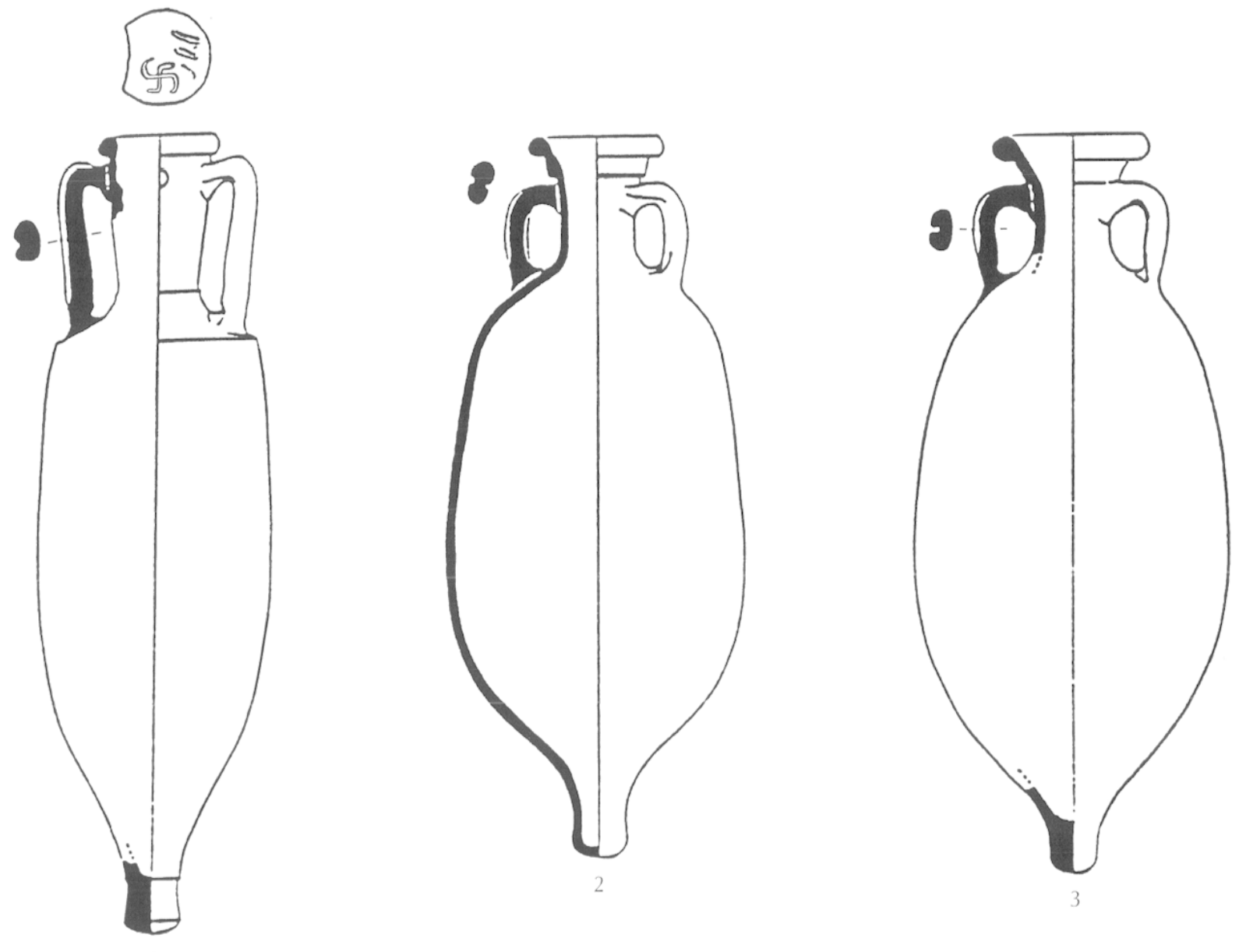

Fig. 18. Les amphores recueillies an XIX's. sur le site de la Mamulention (échelle: $1 / 10$ ).

Tabl. II. Composition chimique moyenne des céramiques communes et des amphores de latelies de la Manutention.

\section{Éléments majeurs en pourcentages d'oxydes}

\begin{tabular}{|c|c|c|c|c|c|c|c|c|c|c|}
\hline $\begin{array}{c}\text { La Manu- } \\
\text { tention } \\
(\mathbf{n = 1 7 )}\end{array}$ & $\mathrm{Na}_{2} \mathrm{O}$ & $\mathrm{K}_{2} \mathrm{O}$ & $\mathrm{MgO}$ & $\mathrm{CaO}$ & $\mathrm{MnO}$ & $\mathrm{Al}_{2} \mathrm{O}_{3}$ & $\mathrm{Fe}_{2} \mathrm{O}_{3}$ & $\mathrm{SiO}_{2}$ & $\mathrm{TiO}_{2}$ & $\mathrm{P}_{2} \mathrm{O}_{5}$ \\
\hline $\mathbf{m}$ & 0,5 & 2,7 & 1,7 & 21 & 0,106 & 12,2 & 4,8 & 56,1 & 0,45 & 0,3 \\
\hline$\sigma$ & 0,1 & 0,1 & 0,2 & 2,5 & 0,01 & 1,1 & 0,4 & 2,1 & 0,04 & 0,1 \\
\hline $\boldsymbol{\sigma} \%$ & 16 & 6 & 11 & 12 & 10 & 9 & 8 & 4 & 8 & 24 \\
\hline
\end{tabular}

\section{Éléments traces en PPM}

\begin{tabular}{|c|r|r|r|r|r|r|r|r|r|r|}
\hline $\begin{array}{c}\text { La Manu- } \\
\text { tention } \\
(\mathbf{n = 1 7})\end{array}$ & $\mathrm{Rb}$ & $\mathrm{Sr}$ & $\mathrm{Ba}$ & $\mathrm{Ni}$ & $\mathbf{Z n}$ & $\mathrm{Cr}$ & $\mathrm{Zr}$ & La & $\mathrm{Ce}$ & $\mathrm{V}$ \\
\hline $\mathrm{m}$ & 120 & 289 & 555 & 65 & 73 & 85 & 104 & 22 & 68 & 79 \\
\hline$\sigma$ & 12 & 33 & 82 & 6 & 8 & 10 & 13 & 13 & 5 & 8 \\
\hline$\sigma \%$ & 10 & 11 & 15 & 9 & 11 & 12 & 13 & 57 & 8 & 10 \\
\hline
\end{tabular}

groupe présente beaucoup de similitude avec celui de la Muette B'.

L'étude granulométrique des inclusions contenues dans la pâte met en évidence une lacune bien marquée entre une fraction sableuse fine et une fraction sableuse grossière. Ceci permet de dire que la phase grossière correspond vaisemblablement à un dégraissant ajouté. Ce dégraissant est constitué de grains de quartz, feldspaths, micas, fragments de roches granitiques et métamorphiques et fragments de rhyolite. Ce cortège minéralogique identique à celui observé dans les céramiques communes de la Muette est caractéristique des sables de la Saône el des alluvions du confluent Saône-Rhône.

1. S. 
I a comparaison de ce matériel avec celui d'habitats comme la rue des Farges ou de dépotoirs comme celui du Bas-de-Loyasse invite à placer l'ensemble du matéricl de cet atelier dans le courant du ${ }^{\mathrm{rr}}$ s. et plus précisément à l'époque Claude-Néron. Ce matériel paraît en effet correspondre à une période de transition entre les productions bien typées de l'époque augustéenne et celles de la seconde moitié du Ir $\mathbf{s}$. C'est le cas pour les mortiers mais aussi pour les amphores. On notera par exemple la présence à la fois des lèvres à ressaut (mais en minorité) et des lèvres évasées et tombantes dont le dépotoir flavien du Bas-de-Loyasse a livé de nombreux exemples (Dangréaux, Desbat, 1988, fig. 18).

Les éléments fournis par le site de la Manutention sont relativement modestes. Ils traduisent cependant l'existence d'ateliers ayant eu une production diversifiće. Les céramiques recueillies en 1966 attestent une production vers le milieu du Irr s., mais on peut supposer que l'activité céramique ne s'est pas limitée à cette période. I.es découvertes du XIX" s., notamment les amphores, laisseraient supposer une phase de production plus ancienne.

\section{Addendum}

Depuis la rédaction de ce chapitre, le site de la Manutention a fait l'objet, en 1997, de sondages préliminaires réalisés par le Service archéologique municipal de Iyon ( ${ }^{\text {mes }} \mathrm{C}$. Becker et M. Monin), dans le cadre d'un projet d'aménagement des anciens bâtiments. Ces sondages, peu profonds, ont révélé, dans les remblais modernes, la présence de nombreux fragments d'amphores lyonnaises de différents types: Dressel 2/4 (type 2B), Dressel 9 similis (types 3A, 3B et 4A) et même une lèvre de Dressel 1.

Ces éléments confirment la présence sur le site même d'une production plus ancienne, d'époque augustéenne. 

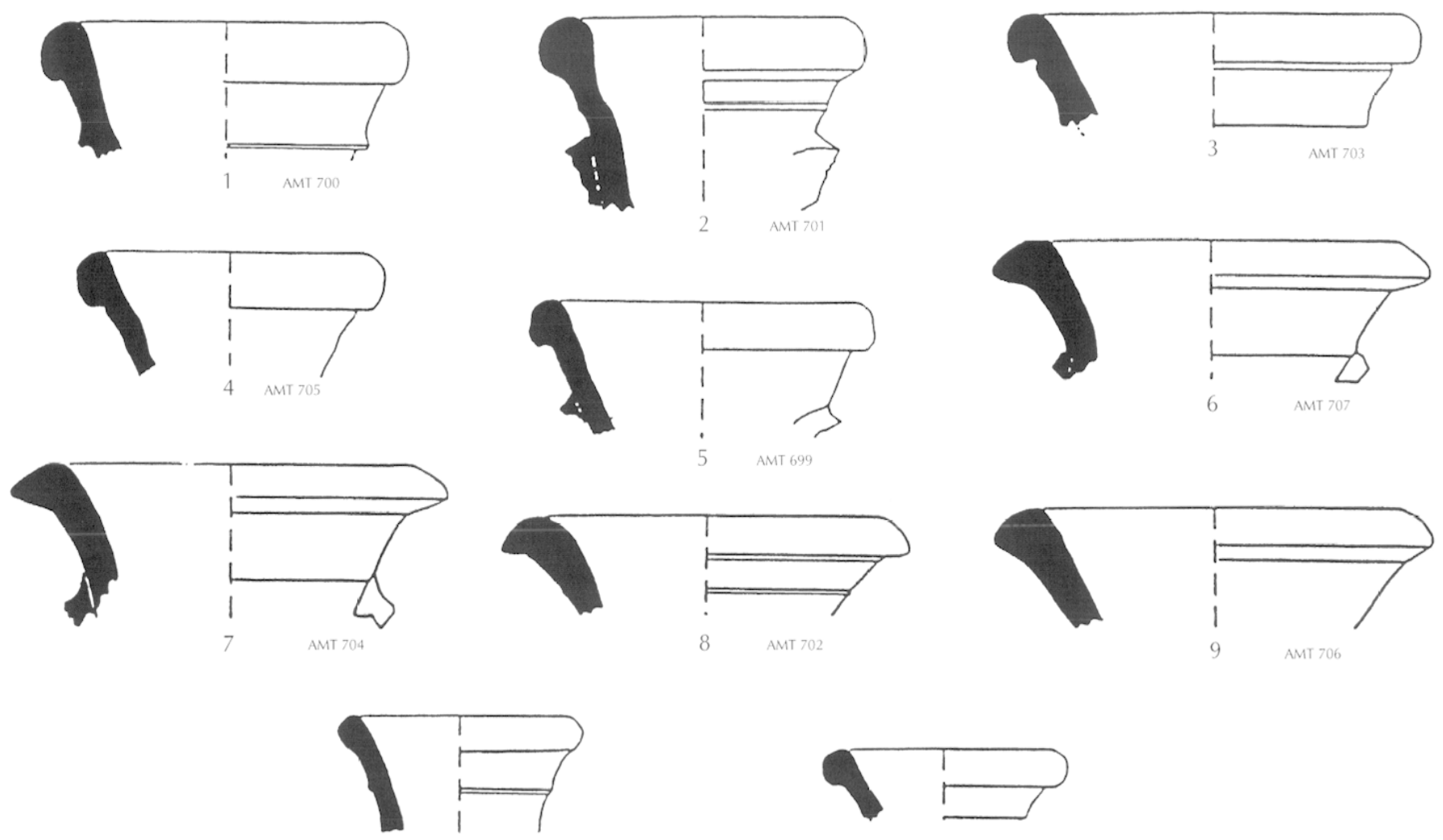

10 AMT 708
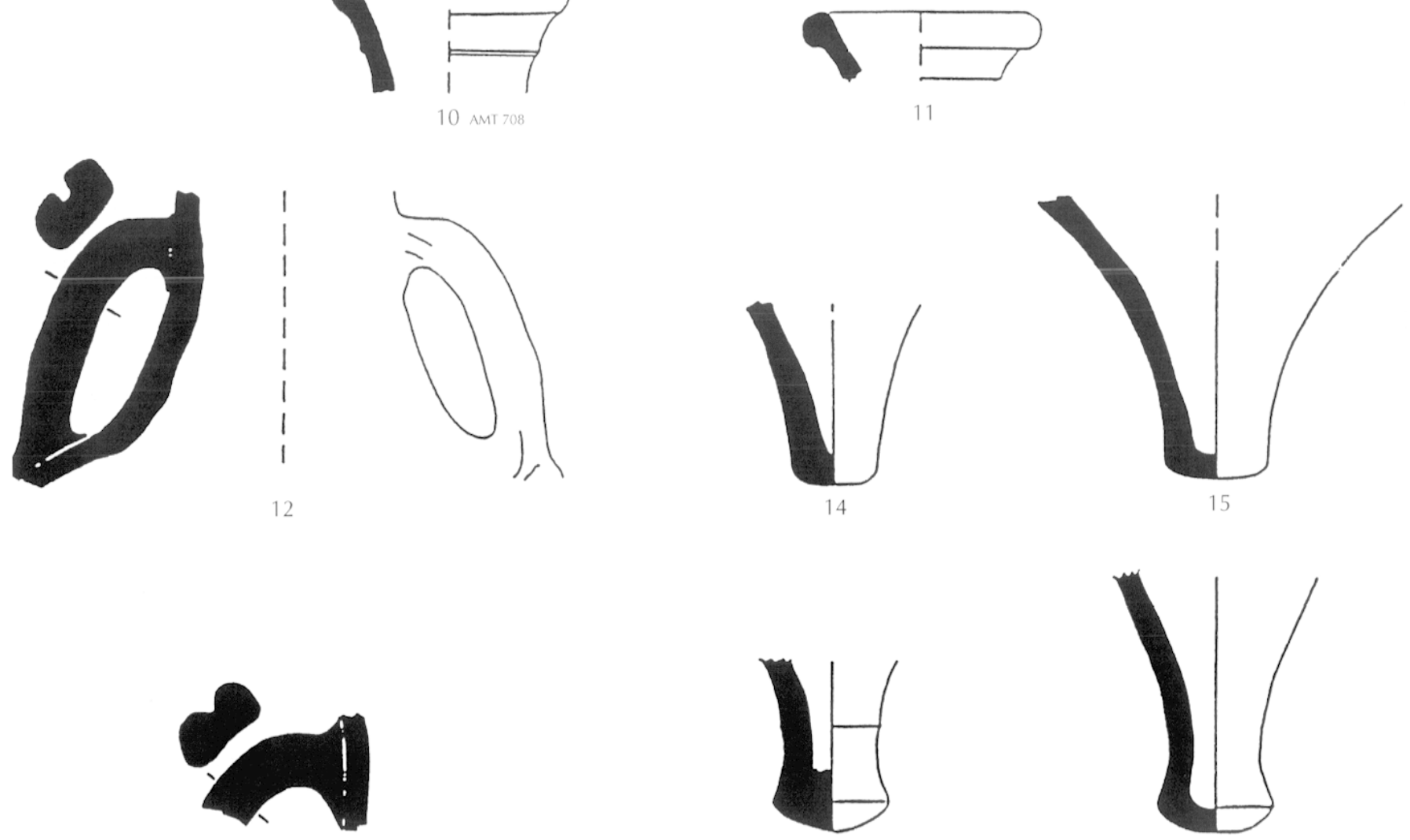

16

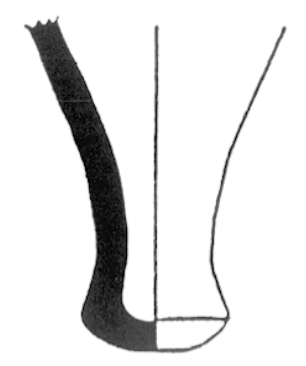

17 AMT 709

P1. 12. Les amphores de l'atelier de la Mamutention (échelle: 1/4). 